\section{Defining shared decision making and concordance: are they one and the same?}

\section{J L Jordan, S J Ellis, R Chambers}

\section{Effective communication is essential}

E ffective communication is an essential element of patient centred medical practice. Terminology has developed to describe different aspects of this interaction between health professionals and patients. While attempting to do research in this field we came across these terms many times and have tried to make sense of them. We are writing this editorial as a guide to those who, like us, have struggled to understand what these terms mean and the differences between them.

The terms "non-compliance" or "nonadherence" have been criticised for suggesting an unequal, paternalistic relationship between health professionals prescribing medication and their patients. To overcome this, the Royal Pharmaceutical Society introduced the model of concordance as a more patient centred approach. ${ }^{1}$ In this model concordance is reached through a therapeutic alliance and negotiation between the prescriber and the patient. The patient is encouraged to discuss concerns about medications that have been prescribed and preferences for treatments and participation in decision making. The health professional gives evidence based information to the patient and shares his or her clinical experience. During the negotiation process compromises are made on both sides and the decision is reached together. If a patient decides to defer the decision, the prescriber makes it for the patient, taking his/her values, beliefs, and concerns into consideration.

Shared decision making is seen as the middle ground between informed choice where decisions are left entirely to the patient and traditional, paternalistic medical decision making. Shared decision making developed in an attempt to reduce uncertainty in the medical community about the most appropriate course of treatment for some conditions. This was accompanied by increased world wide emphasis on patient centred care, leading to the belief that the patient's preferences, rather than the physician's, should dictate disease management. ${ }^{2}$ Shared decision making involves two way information giving (medical and personal) between the clinician and the patient concerning all the options available. The final decision is made jointly with both parties in total agreement. $^{3}$

Shared decision making and creating a therapeutic alliance for concordance increases patient involvement in health

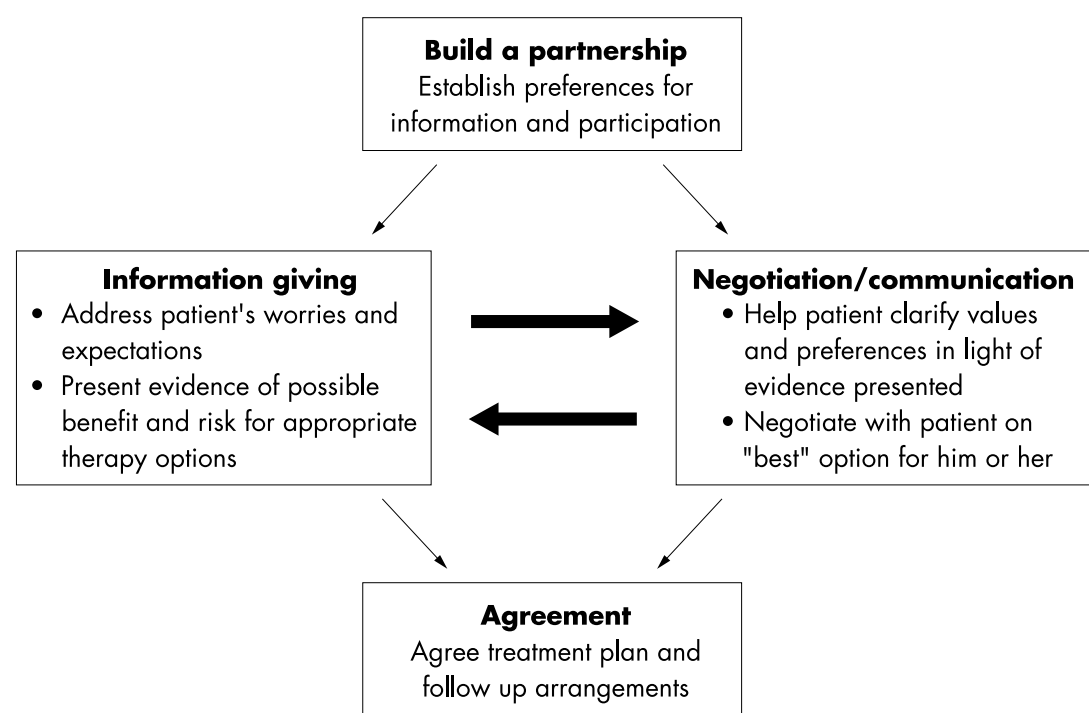

Figure 1 Stages in both concordance and shared decision making consultations. ${ }^{4}$ care decisions and allows a more open exchange between the clinician and the patient. Despite the evolutionary differences the model described for reaching concordance in consultations is the same as the one that has been described for shared decision making. The health professional and the patient have an equal partnership. They share their knowledge and experiences with each other so that an understanding can be reached and a decision about the management of a condition can be made (see fig 1). They both have four common essential elements-partnership, communication (including negotiation), information giving, and agreement.

Building a partnership between the clinician and the patient is the first step towards active patient involvement in reaching concordance or shared decision making. It is the health professional's role to help patients to feel at ease with participating in decisions about their health care. However, a patient may not be comfortable taking on additional responsibility and may want the clinician to decide for him or her. Health professionals must be adaptable and sensitive to the preferences and beliefs of individual patients. Each patient may have different wishes for disease management, the format of information, and particularly their level of participation in making the decision.

Good communication skills are vital to be able to draw out patients' beliefs and preferences about their condition and the medications that they are prescribed. The clinician's views and the clinical evidence have to be expressed clearly and concisely and in the most appropriate format for the patient. The patient needs to understand the information presented to him or her and to be heard and understood by the clinician, for a negotiation to take place. The health professional and the patient have to listen to each other and be prepared to compromise so that an agreement can be reached.

Evidence based information must be useful, acceptable and appropriate for the patient to enable him or her to make a fully informed decision. ${ }^{5}$ This is true for either shared decision making or for concordance in consultations. Evidence based guidelines and summaries of the evidence help to provide this information. However, there are gaps in the evidence and many patient directed information materials are not balanced or omit relevant data. ${ }^{5}$ Health professionals will need the necessary skills and resources to be able to synthesise information for their patients when it is not readily available from other sources.

From the patient's perspective shared decision making and reaching concordance in consultations will feel similar, although very different from the traditional health care encounter that most 
patients are used to. Patients will have to be educated in how to participate in decisions about their health. Whether they are discussing a particular medication that they have difficulty taking or the management of a whole condition, their views and preferences will be sought. Most importantly they are being given an opportunity to have some control, if they wish, over how their health is managed.

The main difference between these terms is that shared decision making terminology encompasses the whole process, whereas concordance is the outcome of that process. There is a danger of taking the word concordance to mean the process of reaching agreement as well as the outcome. This is not necessary as shared decision making already describes this process sufficiently. Concordance between the clinician and the patient should be the goal of all shared decision making encounters. However, an agreement between the health professional and the patient does not always mean that the decision has been shared.

Postgrad Med J 2002;78:383-384

\section{n...................}

\section{Authors' affiliations}

J L Jordan, S J Ellis, R Chambers, Centre for Health Policy and Practice, School of Health,

Staffordshire University, Leek Road,

Stoke-on-Trent ST4 2DF, UK
Correspondence to: $\mathrm{Dr}$ Chambers; r.chambers@staffs.ac.uk

\section{REFERENCES}

1 Royal Pharmaceutical Society of Great Britain. From compliance to concordance. Achieving shared goals in medicine taking. London: RPSGB, 1997.

2 Coulter A. Partnerships with patients: the pros and cons of shared clinical decision-making. J Health Serv Res Policy 1997;2:1 12-21.

3 Charles C, Gafni A, Whelan T.

Decision-making in the physician-patient encounter: revisiting the shared treatment decision-making model. Soc Sci Med 1999:49:651-61.

4 Towle A, Godolphin W. Framework for teaching and learning informed shared decision making. BM 1999;319:766-71.

5 Coulter A, Entwistle V, Gilbert D. Sharing decisions with patients: is the information good enough? BN 1999;318:318-22. 